\title{
RUNX2 RNA interference inhibits the invasion of osteosarcoma
}

\author{
HENG ZENG ${ }^{1}$ and XIAOTAO XU ${ }^{2}$ \\ ${ }^{1}$ Department of Orthopedics, Tongji Hospital, Tongji Medical College, Huazhong University of Science and Technology, \\ Wuhan, Hubei 430030; ${ }^{2}$ Department of Oncology, Renmin Hospital, Wuhan University, Wuhan, Hubei 430060, P.R. China
}

Received April 23, 2014; Accepted January 19, 2015

DOI: $10.3892 / \mathrm{ol} .2015 .3124$

\begin{abstract}
It has previously been demonstrated that the expression of the RUNX2 gene is increased in osteosarcoma tissues or cell lines; however, there is little research available on the effect of RUNX2 on osteosarcoma invasion. In the present study, small interfering (si)RNA to RUNX2 was designed and synthesized, and then transfected into SAOS-2 cells. The effects of RUNX2 RNA interference on the invasion of osteosarcoma cells were detected by the soft agar colony forming test and Transwell ${ }^{\circledR}$ chamber assay. The expression of the associated proteins, vascular endothelial growth factor (VEGF), matrix metalloprotein-2 (MMP-2) and MMP-9, was detected by western blot analysis. The results revealed that the number of cell colonies was reduced dose-dependently by the siRNA and that the number of cells permeating through the filter membrane was decreased following transfection with the siRNA. The inhibition of RUNX2 caused a notable decrease in VEGF, MMP-2 and MMP-9 expression $(0.16 \pm 0.04,0.16 \pm 0.02$ and $0.12 \pm 0.02)$ compared with the empty vector $(0.86 \pm 0.22,0.74 \pm 0.16$ and $0.81 \pm 0.16)$ and blank control $(0.78 \pm 0.12,0.82 \pm 0.18$ and $0.78 \pm 0.14)$ groups, respectively $(\mathrm{P}<0.01)$. It can therefore be concluded that RUNX2 siRNA inhibits the invasion of osteosarcoma cells by inhibiting the expression of VEGF, MMP-2 and MMP-9.
\end{abstract}

\section{Introduction}

Osteosarcoma is a common type of malignant tumor in adolescents and children and thus, its etiology and pathogenesis have previously been unknown. It accounts for $\sim 2.4 \%$ of all malignancies in pediatric patients, and $20 \%$ of all primary bone cancers (1). The estimated annual incidence rate of osteosarcoma in patients $<20$ years of age in the USA is 5.0 per million individuals, and the disease is slightly more

Correspondence to: Professor Xiaotao Xu, Department of Oncology, Renmin Hospital, Wuhan University, 238 Jiefang Street, Wuhan, Hubei 430060, P.R. China

E-mail: xuxiaotao2012@163.com

Key words: RUNX2, osteocarsoma, RNA interference, invasion common in males (5.4 per million individuals) than females (4.0 per million individuals) (1). The prognosis of osteosarcoma is poor. Surgery is the main treatment, however, surgery alone may not prevent metastasis. However, surgery in combination with chemotherapy may prolong patient survival time (2). Certain studies have shown that RNA interference (RNAi) knockdown of cyclo-oxygenase 2 (3), ERK1/2 (4) or relaxin (5) inhibits the growth, invasion and migration of human osteosarcoma cells. The RUNX2 gene plays a crucial role in osteoblast differentiation and bone formation, and is closely correlated with the generation and development of osteosarcoma (6). Studies revealed that the expression of the RUNX2 gene was increased in osteosarcoma tissues or cell lines (7-10), but there is limited research available on the effect of RUNX2 on osteosarcoma invasion. In the present study, RUNX2 was downregulated in the osteosarcoma SAOS-2 cell line by RNAi, and its effects on cell invasion and the potential regulatory mechanism were studied.

\section{Materials and methods}

Experimental materials. The human osteosarcoma SAOS-2 cell line (Cell Bank of the Chinese Academy of Sciences, Shanghai, China) was stored in liquid nitrogen in the laboratory (Central Laboratory, Tonji Medical College, Wuhan, China). Dulbecco's modified Eagle's medium (DMEM) with $10 \%$ fetal bovine serum (FBS) was purchased from Gibco, Carlsbad, CA, USA. Rabbit anti-human RUNX2 (26 kDa; 1:1,000), matrix metalloprotein-2 (MMP-2; 1:1,000) and MMP-9 (1:1,000), and vascular endothelial growth factor (VEGF; 1:1,000) polyclonal antibodies were purchased from Santa Cruz Biotechnology, Inc. (Santa Cruz, CA, USA). Oligofectamine ${ }^{\mathrm{TM}}$ and RUNX2 siRNA double-stranded oligonucleotides were purchased from Invitrogen (Carlsbad, CA, USA). Transwell ${ }^{\circledR}$ chamber models were obtained from Chemicon (Temecula, CA, USA). Western blot analysis kits were purchased from Wuhan Boster Biological Technology, Ltd., (Wuhan, China).

Construction of RUNX2 siRNA sequence and its transfection into the osteosarcoma SAOS-2 cell line. The two purchased RUNX2 siRNA oligonucleotides were identified with S1 (5'-UAACAGCAGAGGCAUUUCGUAGCUC-3') and S2 (5'-GAGCUACGAAAUGCCUCUGCUGUUA-3') in sequence determination, and were matched with the GenBank human RUNX2 cDNA sequence by Blast contrast. Additionally, the negative control sequence (Sn, UUCUCCGAACGUGUCACG 
UUUGUGC) was formulated and synthesized. Each sequence $(100 \mathrm{nM})$ was transfected into the SAOS-2 cell line $\left(1 \times 10^{5}\right.$ cells $\left./ \mathrm{ml}\right)$ by the Oligofectamine liposome. The cells were divided into five groups: The blank control (ConB), empty vector (ConN), S1 transfection (S1), S2 transfection (S2) and Sn transfection (Sn) groups. There was no difference between the treatments in each group, with the exception that ConB and ConN were transfected with phosphate-buffered saline and empty vector of the same concentration, respectively. Only the most effective siRNA was used in the following study.

Western blot detection of protein expression. Cells in the exponential growth phase were selected with cellular protein extracted by radioimmunoprecipitation assay buffer (Wuhan Boster Biological Technology, Ltd.) and the protein level was determined using the bicinchoninic acid protein extraction kit (AR0146; Wuhan Boster Biological Technology, Ltd.). Protein $(50 \mu \mathrm{g})$ was added with $2 \mathrm{X}$ loading buffer. Subsequent to a 5 -min denaturation at $100^{\circ} \mathrm{C}$, the proteins were separated by SDS-PAGE and then transferred onto a nitrocellulose filter. The filter was incubated with specific antibodies overnight at $4^{\circ} \mathrm{C}$ prior to being incubated with secondary antibodies, followed by enhanced chemiluminescence (Wuhan Boster Biological Technology, Ltd.) staining and autoradiography. Images were put into grayscale analysis using BandScan version 5.0 software (Glyko, Inc., Novato, CA, USA).

Soft agar assay for cell anchorage-independent growth. Cells in the exponential growth phase were suspended $\left(1 \times 10^{3}\right.$ cells $\left./ \mathrm{ml}\right)$. Soft agar $(5 \%)$ and nutritive medium were mixed at 1:9, agitated, added to the culture dish and coagulated at room temperature. Cell suspension $(1.5 \mathrm{ml})$ was mixed with an equivalent volume of 5\% soft agar, agitated and placed into the dish. Cell colony formation was observed following 2 weeks of culture at $37^{\circ} \mathrm{C}$ in air containing $5 \% \mathrm{CO}_{2}$. The rate of colony formation (\%) was calculated based on the following formula: Colony formation rate $=($ number of colonies $/$ number of cells incubated $) \times 100$.

In vitro invasion assay. A Transwell chamber (pore size, $5 \mu \mathrm{m}$ ) and 24-well culture plates were purchased from Corning Incorporated (Corning, NY, USA). DMEM without and with $10 \%$ FBS was added to the top and bottom layers of the Transwell chamber, respectively. The cell suspension was adjusted to $1 \times 105$ cells $/ \mathrm{ml}, 50 \mu \mathrm{l}$ of which was added to the upper chamber of the Transwell. At $24 \mathrm{~h}$ post-incubation, the cells adhering to the interior of the chamber were collected and fixed using $10 \%$ formalin, followed by Giemsa dyeing. The cells that had migrated through the membrane were counted. The membrane penetration rate $=$ (number of cells penetrated $/$ number of cells added to the upper chamber) $\mathrm{x} 100$.

Statistical analysis. Data are expressed as the mean \pm standard deviation, and t-tests were performed using SPSS 16.0 software (SPSS, Inc., Chicago, IL, USA). P $<0.05$ was considered to indicate a statistically significant difference.

\section{Results}

Silencing effect of siRNA on RUNX2 protein. RUNX2 siRNA $(100 \mathrm{nM})$ was transfected to the SAOS-2 cell line and western

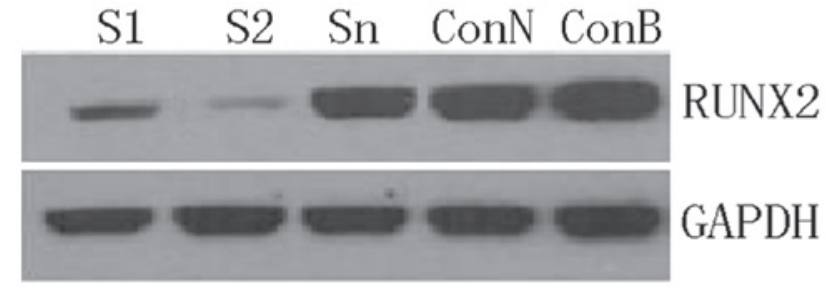

Figure 1. small interfering (si)RNA suppresses RUNX2 protein expression in the SAOS-2 cell line. RUNX2 exhibited high expression levels in ConN, ConB and $\mathrm{Sn}$. Following siRNA transfection, RUNX2 was significantly downregulated in S1 and S2, but particularly S2.

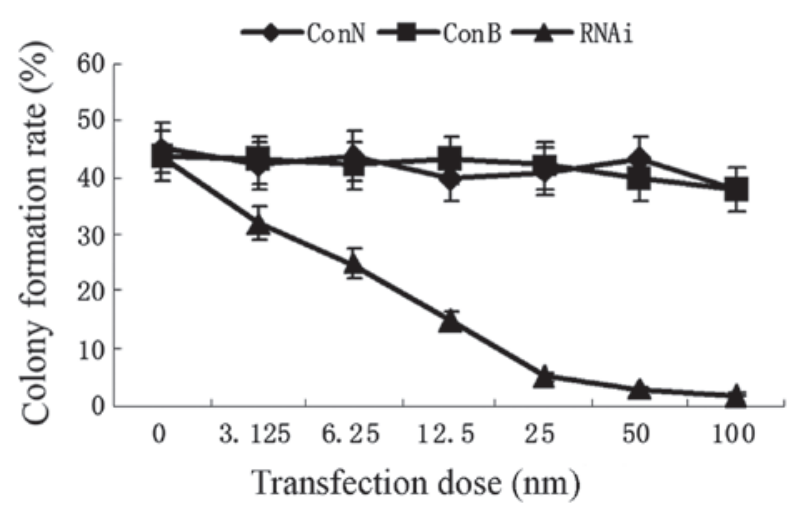

Figure 2. Cell colony formation rate is decreased gradually with increasing S2 small interfering RNA concentration $(0,3.125,6.25,12.5,25,50$ and $100 \mathrm{nM}$ ) in the SAOS-2 cell line.

blot analysis was used to detect RUNX2 protein expression. RUNX2 protein expression in Sn, ConN and ConB demonstrated a grayscale ratio (RUNX2/GAPDH) of $1.26 \pm 0.21$, $1.32 \pm 0.23$ and $1.34 \pm 0.26$, respectively, with no significant differences in the three groups $(\mathrm{P}>0.05)$. RUNX2 protein in $\mathrm{S} 1$ and $\mathrm{S} 2$ exhibited a lower expression level (grayscale ratios of $0.25 \pm 0.04$ and $0.12 \pm 0.02$, respectively) compared with that of the other three control groups $(\mathrm{P}<0.01)$, particularly in S2, in which the RUNX2 level decreased by almost $91 \%$ compared with the control groups (Fig. 1).

Effect of RUNX2 siRNA on the anchorage-independent growth of SAOS-2. Based on the finding that $\mathrm{S} 2$ exhibited the highest efficacy in interfering with RUNX2, S2 was used as the RUNX2-specific interference sequence and set as the RNAi group. Soft agar assay revealed that SAOS-2 formed colonies spontaneously in an in vitro culture system. The cell colony formation rate was decreased gradually with the increasing S2 siRNA concentration $(0,3.125,6.25,12.5,25$, 50 and $100 \mathrm{nM}$ ), while the colony formation rate in the ConN and ConB groups demonstrated only small changes (Fig. 2).

Effect of RUNX2 interference on SAOS-2 invasion. A Transwell chamber was employed to detect the invasion ability of SAOS-2 at $48 \mathrm{~h}$ after S2 siRNA transfection at various concentrations. The results revealed that RUNX2 interference decreased the percentage of membrane-permeating cells significantly compared with the control group (Fig. 3). Moreover, the cell membrane-penetrating rate was decreased gradually with the increasing siRNA concentration (Fig. 4). 


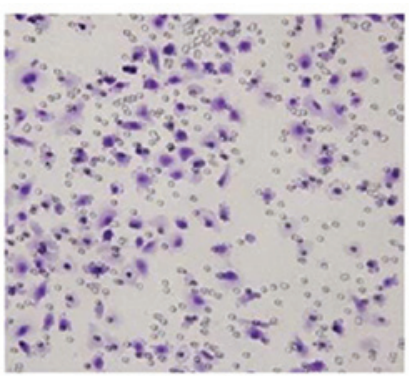

ConN

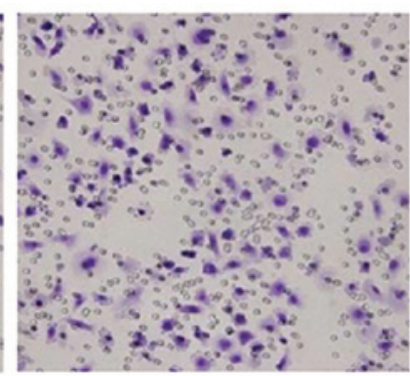

ConB

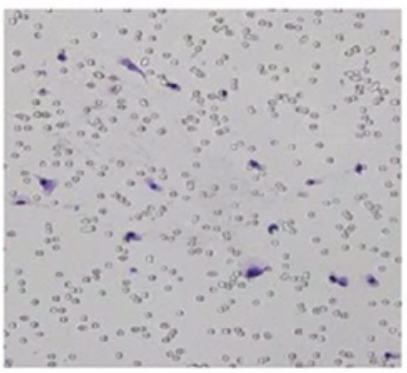

RNAi

Figure 3. Decrease in membrane-penetrating cells following RUNX2 RNA interference in the SAOS-2 cell line.

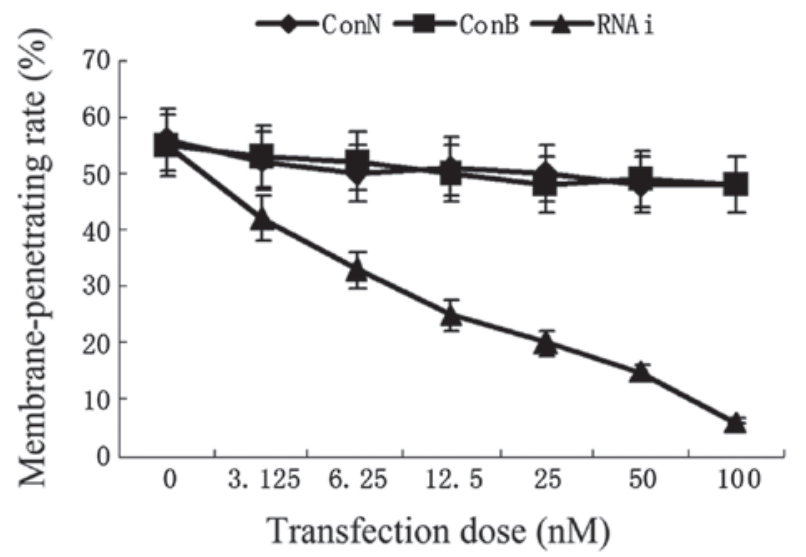

Figure 4. Cell membrane-penetrating rate is decreased gradually with the increasing small interfering RNA concentration in the SAOS-2 cell line.

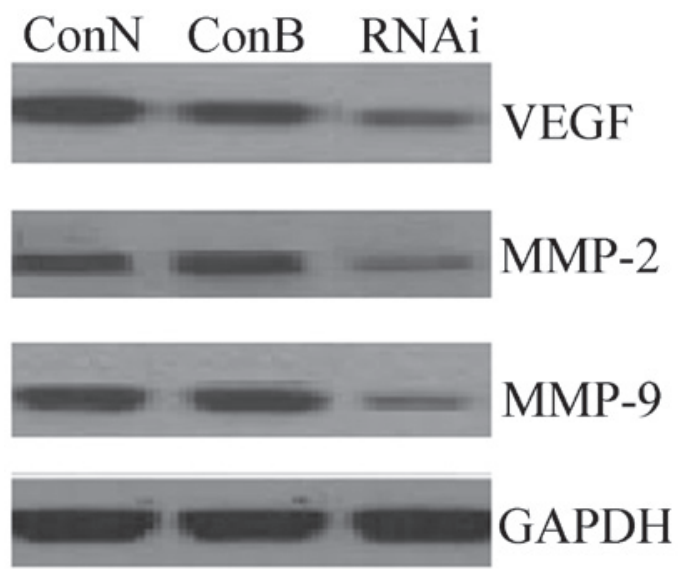

Figure 5. RUNX2 RNA interference downregulates the expression of vascular endothelial growth factor (VEGF) and matrix metalloprotein-2 (MMP-2) and MMP-9 proteins.

Effect of RUNX2 interference on VEGF, MMP-2 and $M M P-9$ proteins. In an attempt to elucidate the mechanism of RUNX2 in controlling osteosarcoma cell invasion, changes in VEGF, MMP-2 and MMP-9 expression levels were analyzed following RUNX2 interference. The results revealed that RUNX2 interference decreased VEGF expression significantly $(0.16 \pm 0.04)$ compared with levels in the ConN $(0.86 \pm 0.22)$ and ConB $(0.78 \pm 0.12)$ groups $(\mathrm{P}<0.01)$. Levels of basal metalloproteinase family members MMP-2 and MMP-9 decreased significantly in the interference group $(0.16 \pm 0.02$ and $0.12 \pm 0.02$, respectively) compared with the ConN $(0.74 \pm 0.16$ and $0.82 \pm 0.18)$ and $\mathrm{ConB}(0.81 \pm 0.16$ and $0.78 \pm 0.14)$ groups $(\mathrm{P}<0.01)$ (Fig. 5).

\section{Discussion}

RUNX2 is a member of the RUNX family. As an osteoblast-specific transcriptional factor, it plays a dominant role in the growth and differentiation of osteoblasts and bone formation, and suppresses preosteoblast proliferation. RUNX2 expression is balanced in normal osteocytes, while it is highly expressed in osteosarcoma cells and forces their overgrowth, playing a similar role to oncogenes (11). It was previously reported that RUNX2 was upregulated in osteosarcoma tissues and that RUNX2 expression was associated with the clinical stage, metastasis and prognosis of the tumor (12). Other studies reported the correlation of RUNX2 expression with the clinical stage and progress of prostatic carcinoma (13) and thyroid cancer $(14,15)$. The role and mechanism of RUNX2 in controlling osteosarcoma formation has previously been unknown, but it was reported that regulating RUNX2 expression could vary the local internal environment of osteocytes, affect osteosarcoma maturity, control apoptosis and matrix metalloproteinase activity and thereby affect osteosarcoma formation (16). It was demonstrated that downregulating RUNX2 could impair the activity of alkaline phosphatase and the deposition of calcium phosphate, and suppress osteosarcoma formation (17).

In the present study, the knockdown of RUNX2 using the RNAi technique was employed to observe the effect of the downregulation of RUNX2 on the invasion ability of osteosarcoma. As a result, RUNX2-specific siRNA suppressed the expression of RUNX2 and achieved RUNX2 gene silencing after being transfected to SAOS-2 cells mediated by liposome (Fig. 1). Initially, a soft agar colony formation assay was performed to detect cell anchorage-independent growth. Anchorage dependence refers to cells that have to anchor with a given matrix to suppress apoptosis and survive. Tumor cells, on the other hand, would remain capable of growing in the absence of anchorage dependence (18). The soft agar colony assay is able to determine the anchorage-independent growth of tumor cells, as well as the malignancy, and the stronger invasion capacity of tumor cells is associated with a greater number of cell colonies (19). The present study revealed that RUNX2-specific siRNA suppresses the colony formation of SAOS-2 in soft agar in a concentration-dependent manner (Fig. 2). The migration and 
invasion capacity of the tumors is correlated with the microenvironment for growth and the extracellular matrix (ECM). A Transwell chamber that imitates such an ECM is a reliable method of assaying the cell invasion ability (20). In the present study, following RUNX2 interference, the number of cells that passed through the Transwell were decreased significantly, and this decrease appeared to be siRNA concentration-dependent (Fig. 3). Preliminarily, this suggests that the interference of RUNX2 suppresses the invasion ability of SAOS-2.

The invasion and migration ability of tumors is strongly associated with their capacity to induce the production of proteinase, which is able to degrade the ECM and basement membrane (21). VEGF, which controls tumor vascularization, plays a key role in tumor formation, invasion and metastasis (22). However, the present study reveals that interfering with RUNX2 suppresses the invasion of SAOS-2 cells, while it decreases VEGF activity. MMPs are another type of molecule associated with tumor invasion. Among them, MMP-9 and MMP-2 regulate the activity of vascular endothelia cells and induce neovascularization. It has been demonstrated that RUNX2 controls the activity of MMP-13 in osteosarcoma cells $(23,24)$, but the role of RUNX2 in MMP-2 and MMP-9 has not yet been reported. The findings of the present study indicate that RUNX2 suppression may inhibit the activity of MMP-2 and MMP-9, thus suppressing cell invasion.

In conclusion, RUNX2 RNAi attenuates the invasion ability of the osteosarcoma SAOS-2 cell line, and this may be attributed to the suppression of VEGF, MMP-2 and MMP-9. For this reason, RUNX2 is of crucial significance in regulating invasion and metastasis in osteosarcoma and is expected to become the targeted molecular therapy for osteosarcoma.

\section{References}

1. Ottaviani G and Jaffe N: The epidemiology of osteosarcoma. Cancer Treat Res 152: 3-13, 2009.

2. Berg J: Canine osteosarcoma: amputation and chemotherapy. Vet Clin North Am Small Anim Pract 26: 111-121, 1996.

3. Zhao Q, Wang C, Zhu J, et al: RNAi-mediated knockdown of cyclooxygenase 2 inhibits the growth, invasion and migration of SaOS2 human osteosarcoma cells: a case control study. J Exp Clin Cancer Res 30: 26, 2011.

4. Si H, Peng C, Li J, et al: RNAi-mediated knockdown of ERK1/2 inhibits cell proliferation and invasion and increases chemosensitivity to cisplatin in human osteosarcoma U2-OS cells in vitro. Int J Oncol 40: 1291-1297, 2012.

5. Ma JF, Liu L, Yang WJ, et al: RNAi-mediated knockdown of relaxin decreases in vitro proliferation and invasiveness of osteosarcoma MG-63 cells by inhibition of MMP-9. Eur Rev Med Pharmacol Sci 17: 1102-1109, 2013.
6. Martin JW, Zielenska M, Stein GS, et al: The role of RUNX2 in osteosarcoma oncogenesis. Sarcoma 2011: 282745, 2011.

7. Sadikovic B, Thorner P, Chilton-Macneill S, et al: Expression analysis of genes associated with human osteosarcoma tumors shows correlation of RUNX2 overexpression with poor response to chemotherapy. BMC Cancer 10: 202, 2010.

8. Pereira BP, Zhou Y, Gupta A, et al: Runx2, p53 and pRB status as diagnostic parameters for deregulation of osteoblast growth and differentiation in a new pre-chemotherapeutic osteosarcoma cell line (OS1). J Cell Physiol 221: 778-788, 2009.

9. Nathan SS, Pereira BP, Zhou YF, et al: Elevated expression of Runx2 as a key parameter in the etiology of osteosarcoma. Mol Biol Rep 36: 153-158, 2009.

10. Ding M,Lu Y,Abbassi S, et al: Targeting Runx2 expression in hypertrophic chondrocytes impairs endochondral ossification during early skeletal development. J Cell Physio 227: 3446-3456, 2012.

11. Lucero CM, Vega OA, Osorio MM, et al: The cancer-related transcription factor runx 2 modulates cell proliferation in human osteosarcoma cell lines. J Cell Physiol 228: 714-723, 2013.

12. Won KY, Park HR and Park YK: Prognostic implication of immunohistochemical Runx2 expression in osteosarcoma. Tumori 95: 311-316, 2009.

13. Yun SJ, Yoon HY and Bae SC, et al: Transcriptional repression of RUNX2 is associated with aggressive clinicopathological outcomes, whereas nuclear location of the protein is related to metastasis in prostate cancer. Prostate Cancer Prostatic Dis 15: 369-373, 2012.

14. Niu DF, Kondo T, Nakazawa T, et al: Transcription factor Runx2 is a regulator of epithelial-mesenchymal transition and invasion in thyroid carcinomas. Lab Invest 92: 1181-1190, 2012.

15. Dalle CL, Frigo A, Francia G, et al: Runx 2 mRNA expression in the tissue, serum and circulating non-hematopoietic cells of patients with thyroid cancer. J Clin Endocrinol Metab 97: E1249-E1256, 2012

16. Mishra S, Vaughn AD, Devore DI, et al: Delivery of siRNA silencing Runx2 using a multifunctional polymer-lipid nanoparticle inhibits osteogenesis in a cell culture model of heterotopic ossification. Integr Biol (Camb) 4: 1498-1507, 2012.

17. Yu J, Yu J, Rhodes DR, et al: A polycomb repression signature in metastatic prostate cancer predicts cancer outcome. Cancer Res 67: 10657-10663, 2007.

18. Merten OW: Advances in cell culture: anchorage dependence. Philos Trans R Soc Lond B Biol Sci 370: 20140040, 2015.

19. Borowicz S, Van Scoyk M, Avasarala S, et al: The soft agar colony formation assay. J Vis Exp: e51998, 2014.

20. Marshall J: Transwell ${ }^{\circledR}$ invasion assays. Methods Mol Biol 769: 97-110, 2011

21. Duffy MJ, Mc Gowan PM and Gallagher WM: Cancer invasion and metastasis: changing views. J Pathol 214: 283-293, 2008.

22. Yancopoulos GD: Clinical application of therapies targeting VEGF. Cell 143: 13-16, 2010.

23. Pei Y, Harvey A, Yu XP, et al: Differential regulation of cytokine-induced MMP-1 and MMP-13 expression by $\mathrm{p} 38$ kinase inhibitors in human chondrosarcoma cells: potential role of Runx 2 in mediating p38 effects. Osteoarthritis Cartilage 14: 749-758, 2006.

24. Hirata M, Kugimiya F, Fukai A, et al: C/EBP $\beta$ and RUNX2 cooperate to degrade cartilage with MMP-13 as the target and HIF- $2 \alpha$ as the inducer in chondrocytes. Hum Mol Genet 21: 1111-1123, 2012. 